$61 \mid 2013$

Vivre le sable !

\title{
Vivre le sable : une introduction
}

"Experiencing Sand" : an introduction

\section{Sébastien Boulay et Marie-Luce Gélard}

\section{OpenEdition}

Journals

Édition électronique

URL : https://journals.openedition.org/tc/7196

DOI : $10.4000 /$ tc. 7196

ISSN : 1952-420X

\section{Éditeur}

Éditions de l'EHESS

\section{Édition imprimée}

Date de publication : 15 décembre 2013

Pagination : 10-27

ISBN : 978-2-7351-1654-6

ISSN : 0248-6016

\section{Référence électronique}

Sébastien Boulay et Marie-Luce Gélard, «Vivre le sable : une introduction », Techniques \& Culture [En ligne], 61 | 2013, mis en ligne le 15 décembre 2015, consulté le 29 septembre 2022. URL : http:// journals.openedition.org/tc/7196; DOI : https://doi.org/10.4000/tc.7196 


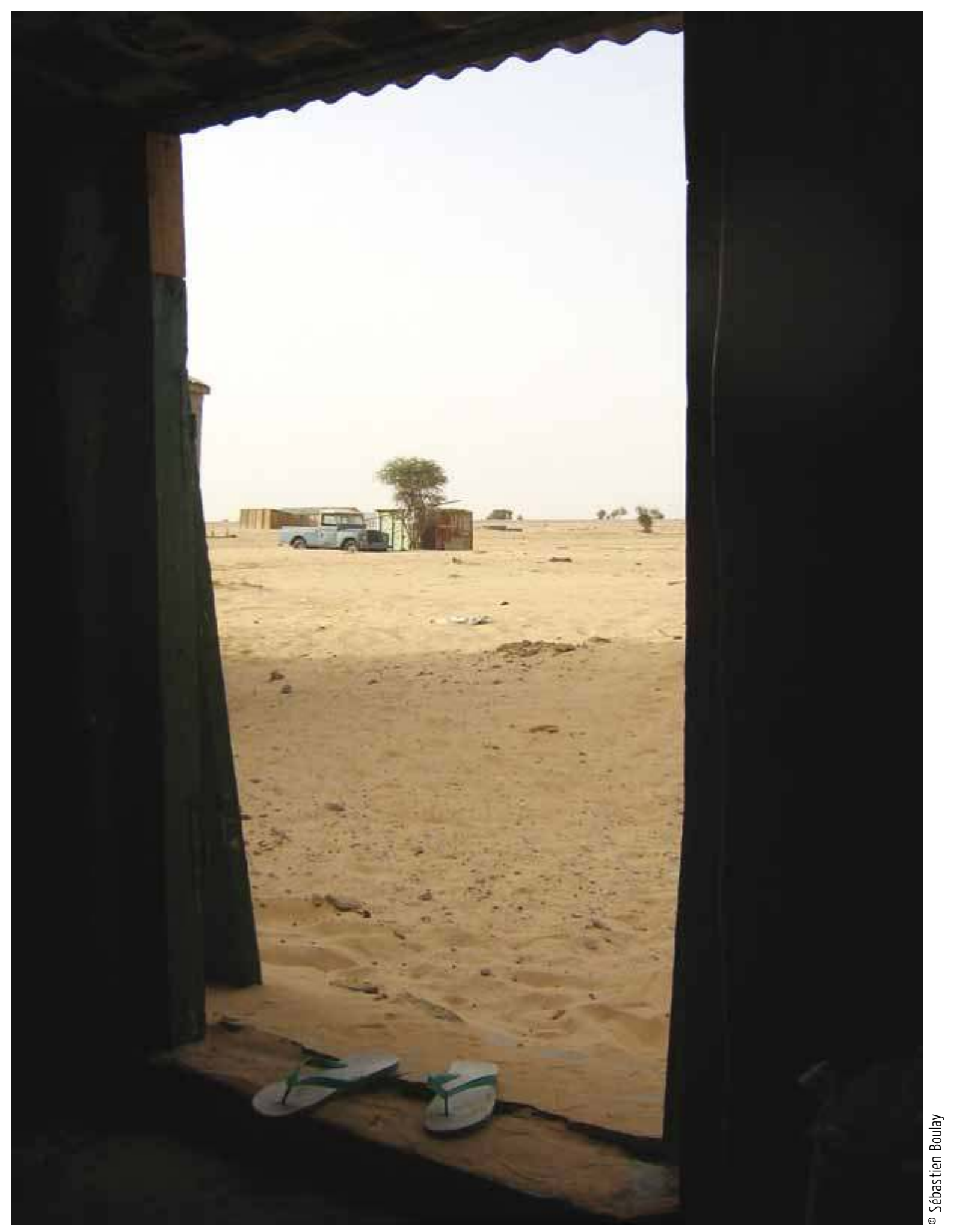




\section{VIVRE LE SABLE: UNE INTRODUCTION}

L'intérêt des sciences sociales pour l'étude des représentations relatives au sable s'est surtout focalisé sur les imaginaires associés aux déserts, lesquels exercent une fascination pérenne. Les récits des "explorateurs" (Foucauld de 1888, 1986, 1998; Thesiger 1959; Monod 1937, etc.), des orientalistes (Pouillon 2006), des administrateurs coloniaux (Caratini 2009) ou des Saint-Simoniens ${ }^{1}$ (Fromentin 1857; Enfantin 1843; Duveyrier 1863, 1864; Urbain 1860) en sont des exemples frappants (Casajus 2006, 2007 et 2009). En effet, ces écrits n'ont pas abordé le thème du sable sinon de manière anecdotique. C'est le sens commun, paradoxalement, qui va voir se développer des perceptions foisonnantes où abonde le poncif d'une équivalence entre désert et sable. Notons la traduction française de l'ouvrage de W. Thesiger (1959), « Arabian Sands » devenu « Le Désert des Déserts » (1978). Le récit de W. Thesiger est, de ce point de vue, sans ambiguité, car le Désert des Déserts est nommé « Sables » par les Bédouins eux-mêmes (Thesiger 1978 : 59).

Ce sont les images d'Épinal qui frappent le chercheur lorsqu'il est question du Sahara et de ses formations dunaires: cités aux prises avec l'ensablement, la légendaire Tombouctou, le dessin des arêtes dunaires, les caravanes, l'Homme Bleu, ou encore le fameux « chant des dunes $»$, aujourd'hui objet d'études scientifiques ${ }^{2}$.

Force est de constater l'absence d'une réelle conceptualisation de la place du sable dans les sociétés du désert; ce numéro propose d'apporter, dans le cadre d'une réflexion collective, un éclairage novateur sur ce qui devenait un « non sujet ».

L'ouvrage d'Anne Varichon et de Carlo Roccella (2006), dont l'entreprise monographique et poétique à propos du sable et de ses usages est remarquable, forme une exception notable. Le texte aborde le sable physique, ses propriétés, les sables mouvants, les
À Pierre Bonte, 


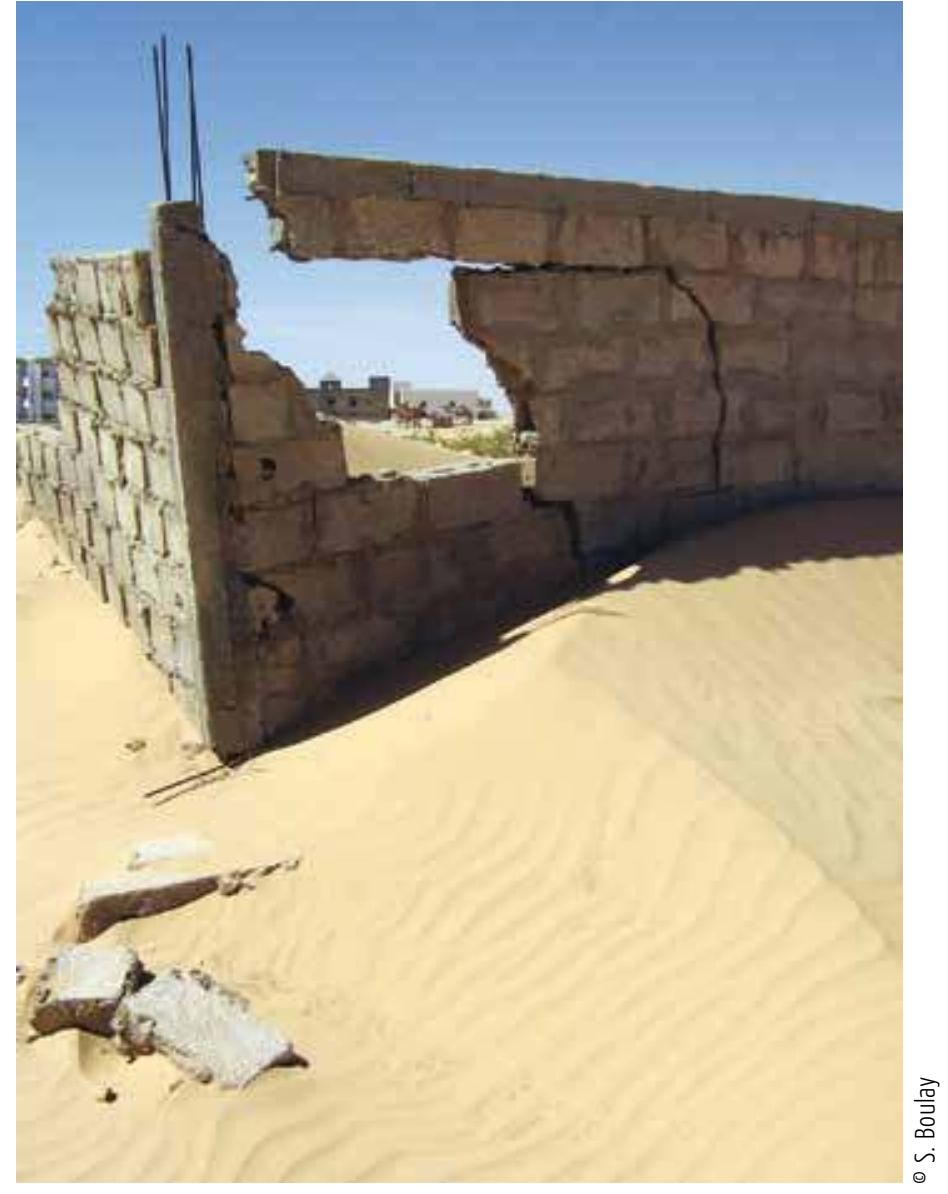

Parpaing et sable, Nouakchott teintes du sable, l'évocation des «bains de sable » au Japon et en Italie, la question du sable comme fluide, solide ou mystique (op. cit. : 54), les aspects utilitaires du sable (sable à maçonner, sable abrasif, moule en sable), le sandboard, la rose des sables, l'arénophilie ${ }^{3}$, etc.

\section{Le sable: matière sans limites, matière impensée?}

Avant d'esquisser quelques pistes de réflexion collective et afin de mieux resituer celles-ci, il est important de savoir à quelle matière on a affaire, quels en sont les propriétés et les contours, et de se demander pourquoi l'anthropologie et plus largement les sciences sociales ne lui ont jamais vraiment accordé d'attention, contrairement à d'autres matières ambiantes comme le vent (Aubert de la Rüe 1940) ou la neige (Therrien 1990, Vadrot 2006).

La définition courante retient à propos du sable l'idée d'un ensemble de petits grains minéraux séparés, généralement de quartz, recouvrant le sol (ReyDebove \& Rey 2013). En fait, le sable se définit avant tout, nous disent les géologues et physiciens, par la taille de ses grains - diamètre inférieur à $2 \mathrm{~mm}$, mais la majorité des grains n'excède pas le millimètre (Welland 2009 : 10) - et non par sa composition ni par sa couleur. Et si les milliards de tonnes de grains de sable qui recouvrent la planète sont majoritairement faits de silice, des grains de sucre ou de sel peuvent « techniquement » être considérés comme du sable, comme on peut s'en rendre compte dans les quelques déserts de sel qui se signalent à différents endroits de la planète. On est face à une matière qui présente une diversité de formes (plus ou moins fin ou grossier, rond ou anguleux) et de couleurs ${ }^{4}$, qui n'est pas inerte comme la roche dont elle provient, mais bien vivante, extrêmement diffuse et mouvante.

À la fois solide, fluide et gazeux, le sable semble rétif aux taxonomies des scientifiques et aux lois de la physique, comme l'explique Ahmed Ould el-Moctar dans ce Thema, à tel point que certains chercheurs ont pensé en faire une matière unique en son genre (Welland 2009 : 32). Liquide quand il est sec, solide lorsqu'il est mouillé, à l'état de poussière sous l'effet du vent, le sable se joue des catégories et a abondamment suscité les réflexions des chercheurs, notamment depuis les travaux fondateurs de Bagnold (1954) sur le mouvement des dunes de sable et la diversité de leurs formes. Compte tenu de ses propriétés, le sable a des effets spécifiques sur les autres matières avec lesquelles il se trouve en contact. Des effets mécaniques tout d'abord: le sable absorbe, sèche, cuit, 
polit, raye. Des effets dynamiques ensuite : le sable saute, se déplace, transporte, charrie, ensevelit ou découvre. Des effets chimiques, enfin, lorsque le sable est mêlé à d'autres matières, comme le ciment dans la construction, les pigments dans les arts plastiques, etc. D'ailleurs, le sable n'est pas sans évoquer immédiatement une autre matière ambiante, l'eau, avec laquelle il partage certaines caractéristiques (liquide, solide, poudreux): il aurait finalement des propriétés physico-chimiques inverses de celles de l'eau (délayer, faire fondre, assouplir) et des propriétés dynamiques semblables (submersion, transport...).

Ces caractéristiques énoncées, à partir desquelles va se décliner une diversité de dénominations, de représentations et d'usages du sable, on peut s'interroger sur les contours de cette matière, en bien des aspects inclassable. Tout à la fois substance, surface, paysage, peut-on qualifier le sable de matière « naturelle » car non transformée, de matière « ambiante » car omniprésente dans nos vies, particulièrement dans les sociétés du désert? Où commence le sable lorsque l'on sait par exemple que dans certaines régions on « fabrique » du sable de couleur en grattant la roche pour obtenir une matière qui sera utilisée dans l'art pariétal ou les peintures de sable (sand-paintings)? Quelles sont les frontières de cette matière lorsqu'elle se confond avec d'autres substances, avec la terre en particulier, - frontières que les langues ne nous aident pas toujours à saisir: dans l'ouest du Sahara par exemple, on utilise le même terme arabe « trâb » pour désigner la terre et le sable.

La singularité de cette matière explique sans doute en partie qu'elle soit restée largement impensée des travaux en anthropologie de la culture matérielle et des techniques, plus préoccupés par la «matérialité » des objets qui nous entourent que par un questionnement plus englobant sur les matières « ambiantes", leurs propriétés et leurs interactions avec les sujets et les objets. Les réflexions de M. Mauss sur les techniques du corps (1936) n'ont ainsi pas pris en compte les matières sur lesquelles prenaient appui les conduites motrices qu'il décrivait. Et si A. Leroi-Gourhan a intitulé le premier de ses deux volumes sur la classification des techniques et les problèmes d'évolution technologique L'homme et la matière (1945/1992), c'est avant tout parce que selon lui « c'est la matière qui conditionne toute technique et non pas les moyens ou les forces » (1992: 19) et afin de classer les modalités de l'activité technique selon la nature des matières disponibles, et non pour s'interroger sur ce

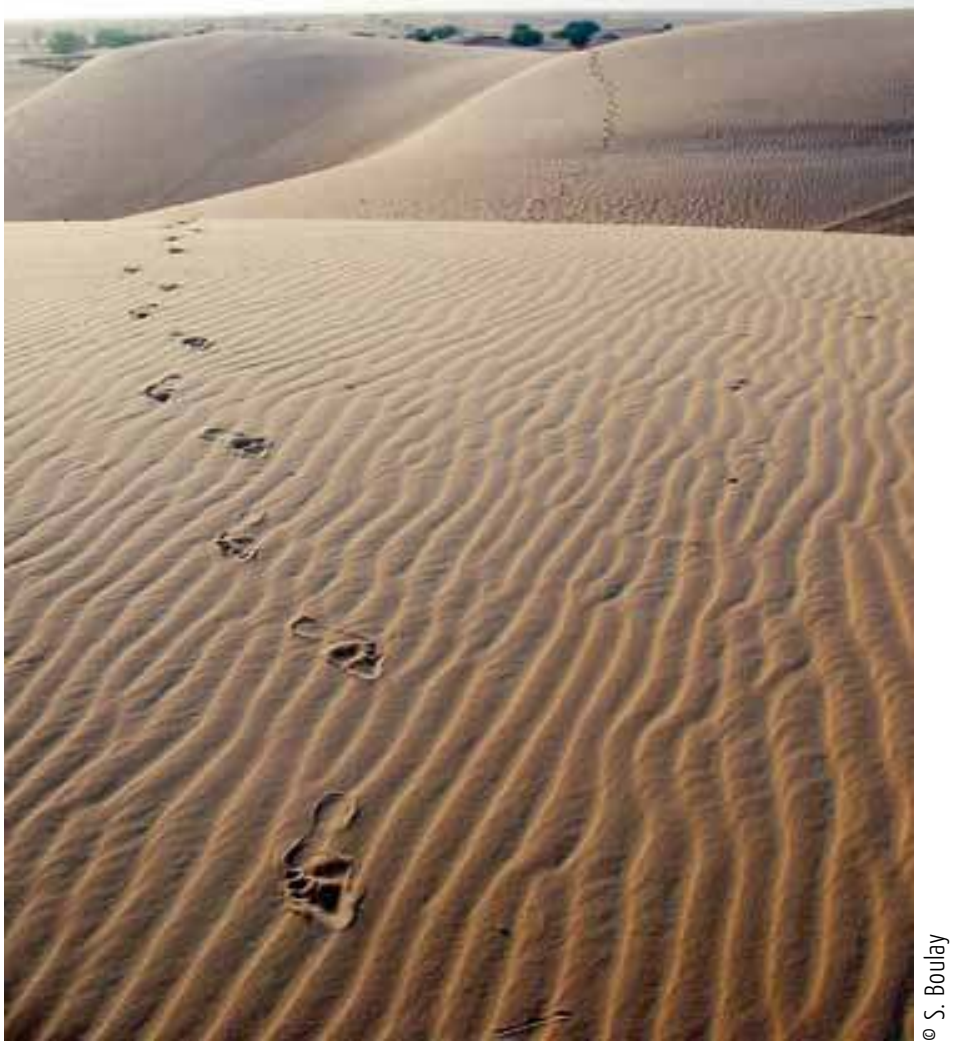


Descente de dune

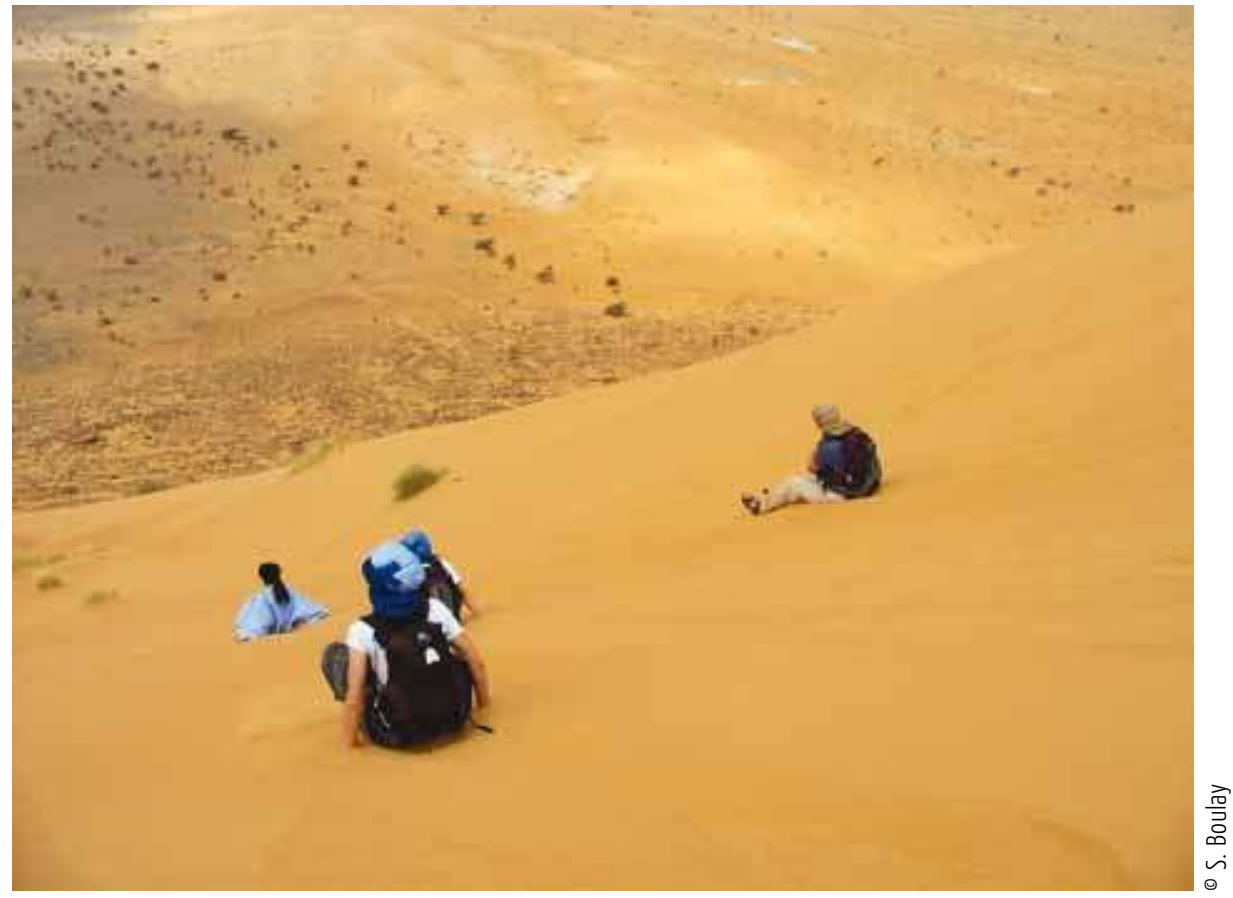

que « fait » une matière comme le sable aux individus. Lanthropologie a proposé une somme imp<ortante de travaux sur la production et la consommation d'objets et, plus récemment, sur le rapport entre sujets et objets, entre corps et artefacts (Warnier 1999, et Julien \& Warnier 1999). Mais il semble que l'on ait oublié en route le corps à corps avec ces matières « ambiantes » qui nous entourent, dans lesquelles certaines sociétés, comme celles qui seront à l'honneur dans ce Thema, « baignent » au quotidien.

Dans un texte récent, Tim Ingold (2007), s'inspirant notamment des travaux pionniers du psychologue américain J.J. Gibson (1979), nous rappelle pourtant que ces matières ambiantes sont les fondations sur lesquelles et grâce auxquelles notre vie quotidienne et nos actions peuvent se dérouler. Ingold constate que beaucoup d'anthropologues considéraient que tout ce qui était « matériel » résidait dans les choses/objets, comme si tout le reste relevait de l'immatériel. Or, il rappelle que la matérialité ne commence pas avec les objets ni ne se résume à ceux-ci: sujets et objets sont matière et se meuvent dans (de) la matière (air, eau, sable, béton...), et parmi d'autres matières. Et de remarquer qu'aucun objet n'est éternel alors que l'est la matière dont ils sont faits. Pour Ingold, les matières se transforment et nous transforment; elles sont " processuelles et relationnelles » (Ingold $2007: 14$, notre traduction).

Ce Thema pourra alimenter les réflexions sur ces questions, car il aborde des sociétés qui vivent cette matière au quotidien. Aujourd'hui, près d'un milliard d'individus évoluent dans des régions arides (moins de $200 \mathrm{~mm}$ de précipitations/an) ou hyper-arides (moins de $25 \mathrm{~mm} / \mathrm{an}$ ), qui couvrent $20 \%$ des terres émergées de la planète, dont $20 \%$ sont sableuses (Welland 2009 : 148), avec cependant des déserts plus « dunaires » que d'autres, comme le désert australien évoqué par le texte de Sylvie Poirier. 


\section{Évolutions et involutions du projet}

Notre réflexion fut d'abord restreinte au Sahara, mais il nous a rapidement semblé important d'ouvrir celle-ci à d'autres déserts (Australie notamment). Il convient aussi de noter les aventures que ce projet a suscitées en évoquant rapidement les conditions mêmes de la recherche, car si l'occultation des conditions de production du savoir des ethnologues fait autorité dans le champ scientifique (Kilani 2000 : 38), il permet ici bien au contraire de mieux saisir la construction d'un objet/sujet, ainsi que celle des résultats attendus et des retombées...

S'il ne s'agissait pas de poser la question ontologique des sociétés de désert, laquelle semble clairement définie par les géographes et climatologues, il convenait de chercher à comprendre la place et les perceptions du sable dans ces sociétés. Cette limite thématique écartait de fait les études sur les littoraux, les plages (Devienne 2011) ${ }^{5}$, les coutumes balnéaires et les loisirs (Corbin 1995), tout en notant au passage l'abondante description des pratiques et interactions sur les plages (jeux, vie sociale des plages, etc.) (Augé 1995) qui ne donnait pas pour autant lieu à une interrogation sur le sable.

L'étalon de l'aridité des zones désertiques est celui des seules précipitations, ce qui mériterait une réflexion sur la notion même de désert où le sable et la géomorphologie des lieux seraient sans réelles incidences. En effet les «sols dunaires comptent 80 à $90 \%$ de sables, mais il suffit qu'un sol comprenne $55 \%$ de sables pour que l'on parle de texture sableuse » (Morel 2008 : 56). On sait aussi l'importance des sables cimentés (ripple-marks) qu'étudient les géomorphologues pour comprendre l'histoire des déserts.

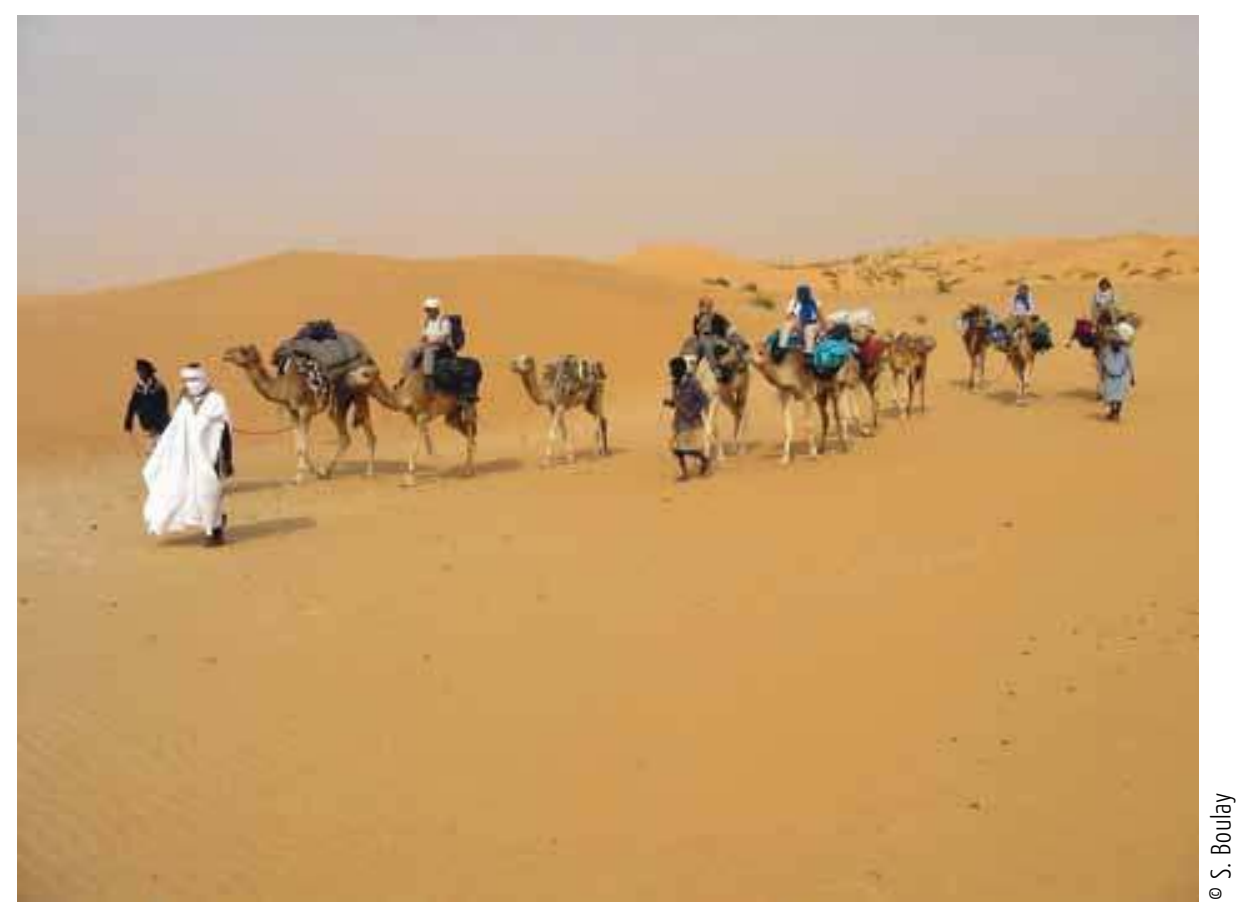

Caravane de trekkeurs, Adrar 
Si les dunes occupent peu l'espace du désert, le sable est présent partout en faible quantité sur l'ensemble du Sahara. Dans ce numéro, les dunes de sable, l'origine du sable et les accumulations éoliennes sont décrites du point de vue physique par Ahmed Ould el-Moctar qui, avant même l'ethnologue, prend la trace sur le sable comme signe... «Chaque direction du vent s'imprime sur le sable en donnant des réseaux extrêmement variés qu'un aéro-dynamicien saura interpréter ». (Soleilhavoup 2010 : 57).

L'étude des usages sociaux du sable est délaissée par la recherche. Ainsi, par exemple, la belle expérience du géographe Marc Côte qui propose une analyse des images satellitaires du Sahara mais où ne figure pas l'entrée thématique « sable ». L'objectif de l'ouvrage est de montrer « les signatures » et les « traces » humaines au Sahara: oasis, agriculture saharienne, ksours, villes mortes, importance de l'argile dans l'architecture saharienne, villes-oasis, eaux usées, salines, bases pétrolières, murs et camps, avec deux évocations seulement du sable, celle des traces des troupeaux et de l'ensablement (2012 : 246-256).

Soulignons que l'avancée du sable peut aussi être positive et le sable devenir l'objet d'une attention spécifique et d'une protection écologique. Ainsi, le minuscule désert de sable dans la province de Manitoba, au Canada, appelé « The Spirit Sands », se trouve aujourd'hui menacé par la végétation alentour.

Si l'appel à contribution initial intitulé « Déserts, sable et techniques » n’a suscité qu'un nombre modeste de réponses et illustre la difficulté pour les sciences humaines et sociales de lier « matière, perceptions et vécus », la question méthodologique s'est avérée d'autant plus stimulante qu'elle a ouvert des pistes de réflexion, des champs d'étude et des questionnements nouveaux. C'est l'interface et la complémentarité d'une analyse matérielle (objet sable) et symbolique (les représentations endogènes du sable) qui constituent l'originalité de cette rencontre d'articles. Ainsi, l'étude du sable dans les sand-paintings (évoqué en Australie par Sylvie Poirier) initie la réflexion sur le sable comme support, signe et symbole. Les peintures acryliques ont remplacé le sable et si ce passage a bien été analysé (l'ouverture vers les marchés de l'art des peintures aborigènes), l'importance et l'usage initial du sable, de la terre ou d'autres matériaux granulaires n’a pas retenu l'attention.

Palmeraie encerclée par le sable

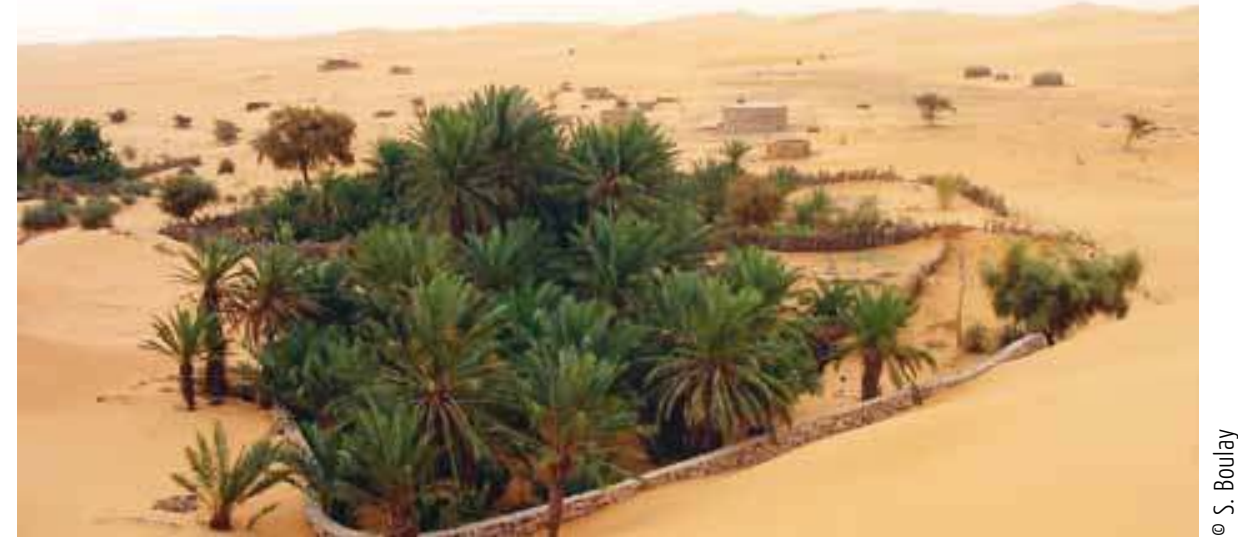




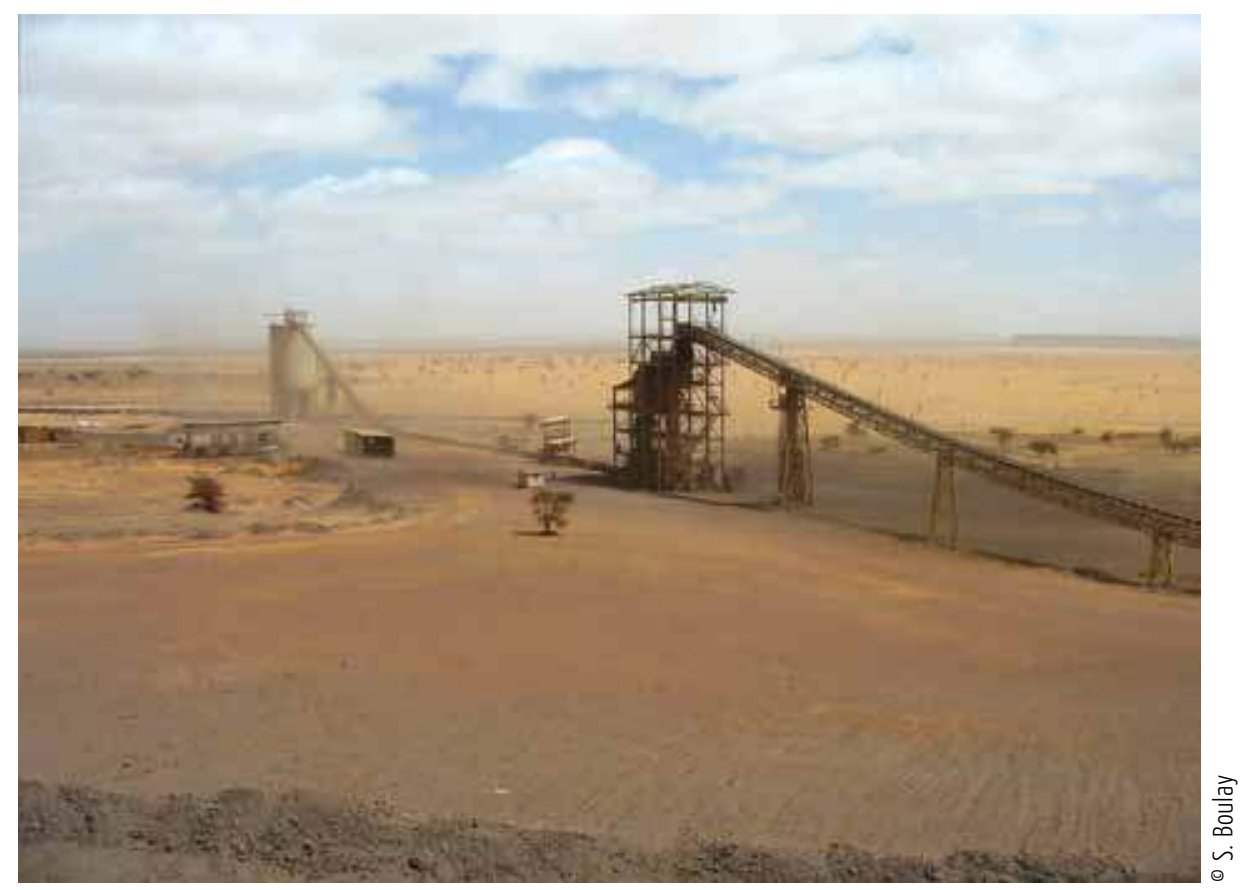

C'est aussi le cas chez les Navajo, qui n'ont pas abandonné le support, mais dont l'ethnographie demeure attachée à l'explicitation du symbolisme des dessins et des rituels qui les accompagnent (Newcomb et Reichard 1975; Bahti et Baatsoslanii 2000) et non à l'analyse du sable comme matière et support d'expression. En effet, le geste et sa maîtrise dans l'utilisation du sable comme fluide tinctorial (substitut graphique) et le caractère volontairement temporaire n'ont pas fait l'objet d'une étude plus approfondie, en dehors des liens entre représentation sacrée et codes comme pour les mandalas tibétains (Varrichon et Roccella 2006 : 58). Les dessins sur le sable sont pourtant nombreux, comme les « sanddrawings » du Vanuatu ${ }^{6}$ ou la divination par le sable. « Les dessins sur le sable sont des tracés linéaires, souvent complexes, ayant des propriétés intéressantes qui relèvent de la théorie des graphes. Cette technique est pratiquée dans plusieurs régions du monde, et consiste simplement à tracer un sillon sur une surface de sable » (Chemillier 2004 : 272). L'exécution d'un dessin se réalise dans un cadre rituel spécifique, narration mythique, évocation du chemin vers le monde des morts, etc. Dans la mesure où les dessins doivent être exécutés par une ligne continue sans lever le doigt, les études se sont orientées vers les modèles mathématiques ${ }^{7}$ qu'ils sous-tendent (Deacon 1934, Ascher 1998, Gerdes 1995) ou sur la fonction symbolique des dessins comme principe narratif (Cabane 1995 et 1997). Pas d'interrogations sur le sable comme matière meuble, élastique et parfaitement adaptée à ces arts graphiques éphémères, voir l'exemple des sands painting réalisés à New-York. Là encore, ce numéro ouvre des pistes et des questionnements.

De la même façon, la recherche sur les géoglyphes qui utilisent l'oxydation des sols du désert (Chili) comme technique d'inscription trouve une résonance singulière dans ses liens avec les pistes caravanières où les géoglyphes deviennent des « marqueurs territoriaux » (B. Clarkson 1999 : 134). L'utilisation des surfaces inclinées des sols ouvre la 
comparaison avec les traces produites par les différentes mobilités au Sahara. Le reg est recouvert de gravillons qui sont noircis par la patine du désert mais le substrat de sable jaune qui se trouve juste dessous crée une marque plus claire. Ces traces sont informatives, elles permettent le déplacement dans des contextes particuliers, comme celui des transports nocturnes sans éclairage, c'est le cas pour les deux roues par exemple, la piste étant rendue très légèrement visible de nuit, par une fine trace blanchâtre.

«Un an après, dix ans après, ces traces peuvent s'observer encore. Car, en l'absence de toute précipitation ou humidité, la surface du sol demeure inchangée. Dans le désert libyque, soixante ans après l'événement de la guerre de 1940, les traces des véhicules de la colonne Leclerc demeurent visibles » (Côte 2012 : 248).

Ces traces (Ginzburg 1989) demeurent largement sous analysées, l'ethnographie des usages du sable reste en effet à conduire: depuis l'examen du jet de sable pour prouver des intentions pacifiques (Thesiger 1978 : 84), à son utilisation pour manifester discrètement et sans parole son attirance (op. cit. : 240-241).

\section{Des cultures matérielles du sable?}

Le titre de ce Thema « Vivre le sable! » soulève d'emblée la question de l'adaptation des sociétés du désert à cette matière. S'adapter ne consiste-t-il pas à la fois à faire face, à affronter, tout en déployant un certain nombre d'attitudes, de techniques et d'objets qui vont

Train minéralier

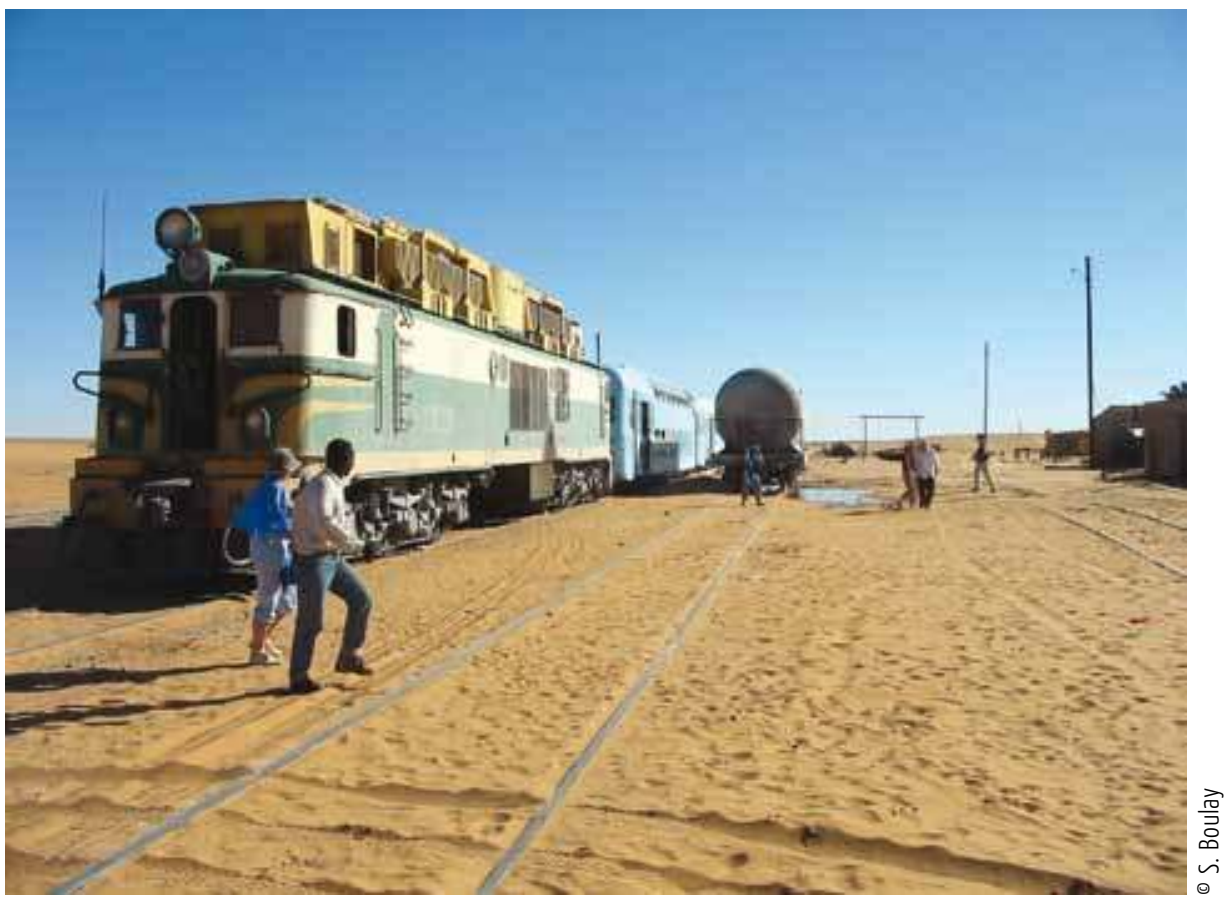




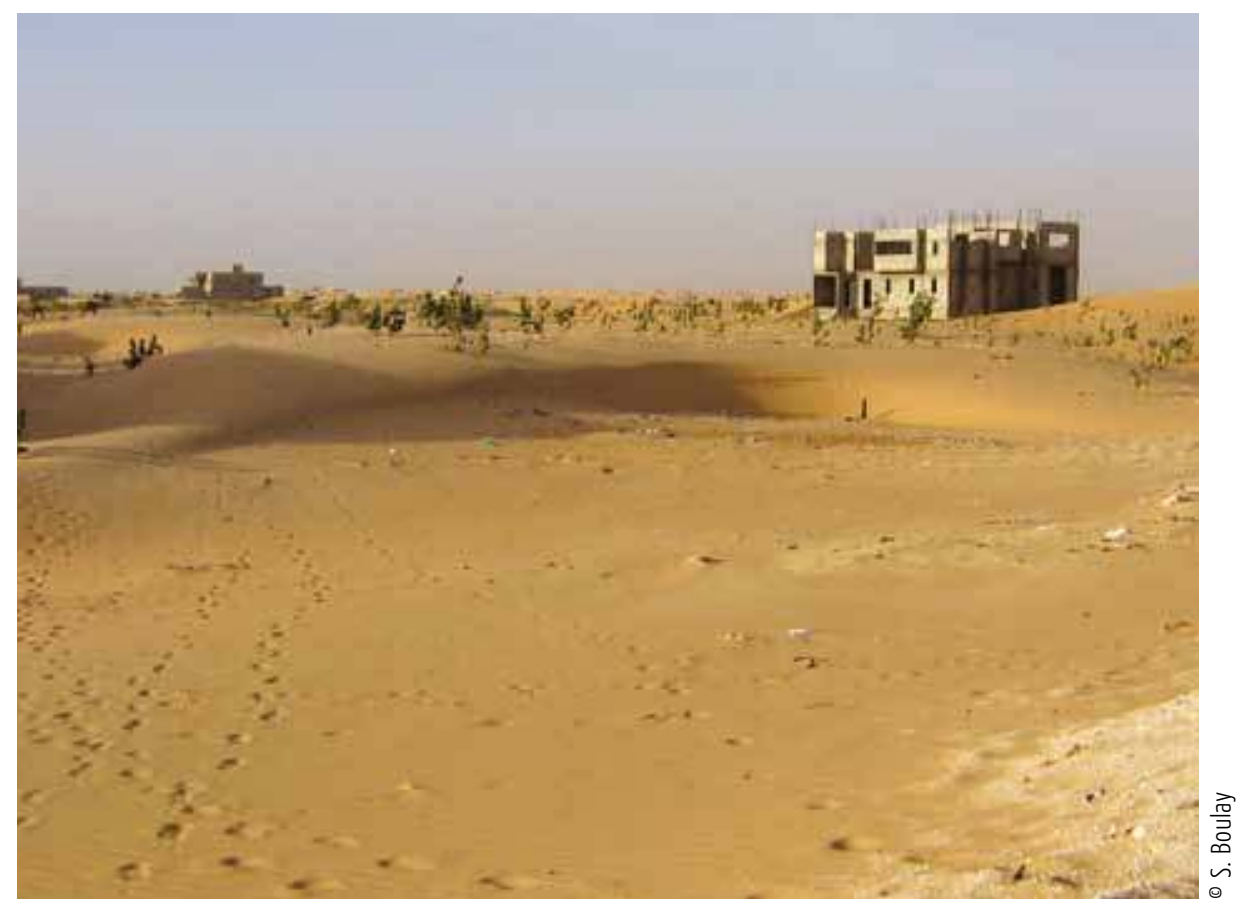

permettre de vivre au mieux (au contact direct de) cette matière? Il est intéressant, à ce titre, de mettre en regard l'adaptabilité humaine et l'adaptabilité animale à ces milieux arides sableux, la seconde semblant avoir une longueur d'avance sur la première si l'on observe, par exemple, les techniques de camouflage et d'habitat des fennecs et autres vipères des sables ou scorpions. Le texte de Bernard Faye s'intéresse à l'animal le plus emblématique du Sahara, caravelle du désert, qui a largement permis à l'homme de survivre dans ces régions, à tout le moins de les traverser: le dromadaire. Il montre à quel point cet animal s'est adapté aux extrêmes qui caractérisent ces contrées (chaleur diurne, forte amplitude thermique entre le jour et la nuit, sécheresse, pauvreté du couvert végétal, distances à parcourir pour s'abreuver); une adaptation qui passe par un métabolisme spécifique lui permettant de tirer le profit maximum des ressources qu'il ingère, mais également par une physionomie particulièrement préparée à affronter les sols et les projections de sable, et par un rôle de régénération de la flore grâce aux déjections qu'il disperse au cours de ses pérégrinations.

Vivre le sable, c'est à la fois subir cette matière, ses intrusions permanentes dans la sphère domestique, mais aussi dans l'intimité physique des personnes, la contourner ou l'éviter, et agir sur elle de la façon la plus efficace et harmonieuse, relativement aux moyens dont on dispose. Agir suppose des choix techniques (Lemonnier 1993) qui soient en concordance avec les représentations que l'on a de la matière et de ses propriétés. Cela suppose également d'habiter le sable d'une certaine manière, en plantant par exemple sa tente selon une orientation qui préserve au mieux ses habitants des assauts du sable, de recourir à des objets et donc à fabriquer des objets garantissant le confort le plus optimal dans ces situations, d'adopter des postures corporelles qui soient les mieux à même de tirer parti des qualités de confort du sable tout en se prémunissant de ses désagréments, de marcher dans le sable d'une certaine manière. Le texte de Sébastien Boulay explore cette quête d'équilibre 


\section{Chamelles parquées dans les nouveaux quartiers résidentiels de Nouakchott}

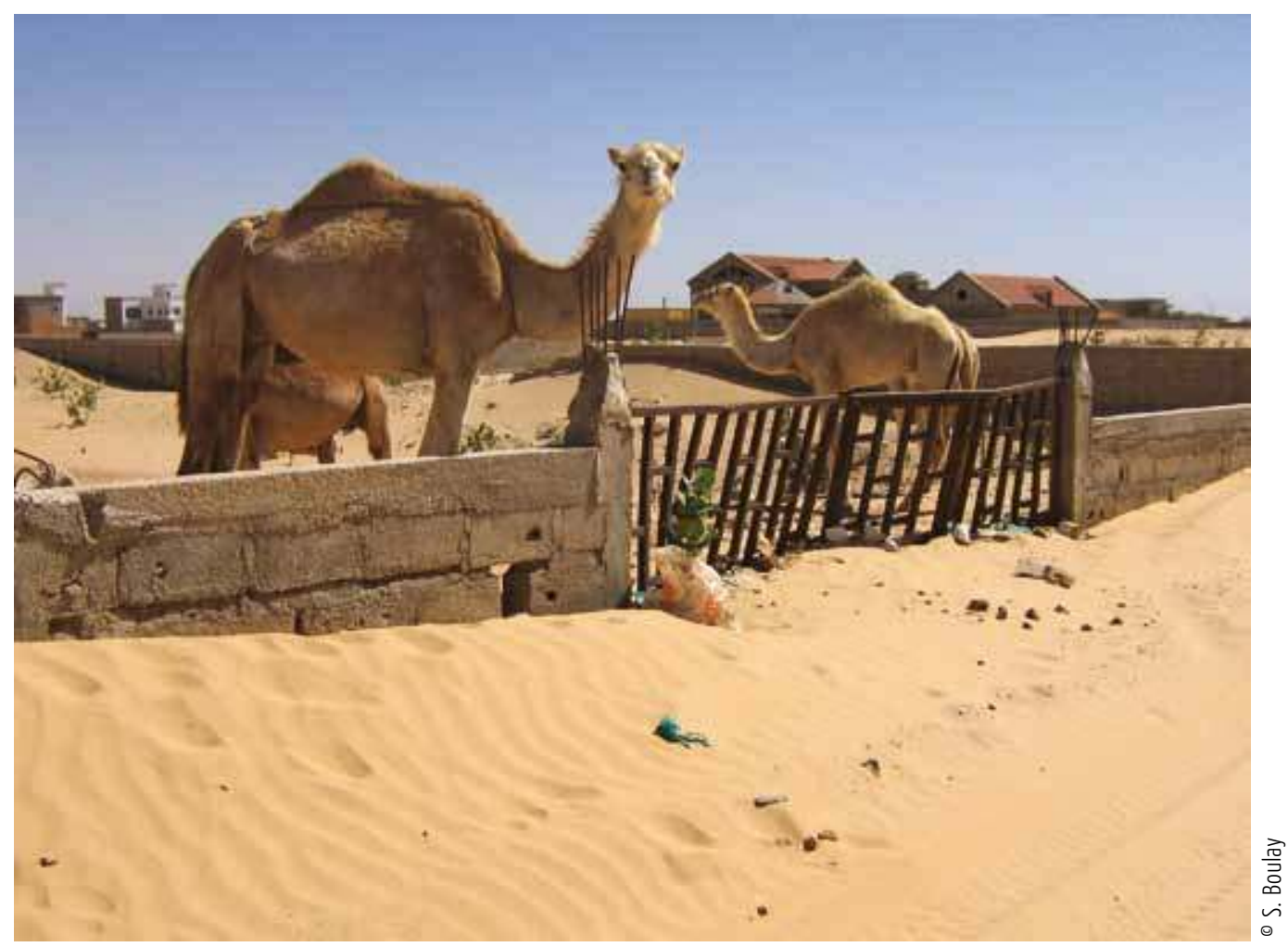

permanente des habitants du désert, et notamment le rôle des objets domestiques, de leurs formes dans cette recherche, tentant au passage de répondre à la question de l'existence d'un « style » (Martinelli 2005) propre à ces cultures matérielles du sable. Loin d'être déterminant et surtout explication unique du style matériel des sociétés du désert, le sable semble toutefois conférer un design particulier ou une importance singulière à certains artefacts.

La créativité des sociétés vivant au contact direct du sable ne s'exprime pas seulement dans la recherche du confort domestique, elle se manifeste dans bien d'autres domaines, à commencer par ceux mobilisant des techniques d'enfouissement. Enfouissement du corps d'un défunt, ainsi que l'évoque l'entretien avec Robert Vernet (Curiosa) qui aborde longuement les pratiques d'inhumation des populations néolithiques de l'Ouest saharien, dont certains vestiges nous sont parvenus, grâce à l'aridité de ces régions et grâce au pouvoir de dessiccation du sable. Enfouissement également à des fins thérapeutiques de corps en quête des bienfaits du sable brûlant de la part de citadins qui pratiquent les « bains de sable » en plein été à Merzouga, analysés par Marie-Luce Gélard. S'enterrer dans le sable est aussi une technique de camouflage employée au Sahara dans des situations de guerre et de protection contre un ennemi. Cette technique d'immersion est également couramment employée pour sécher des aliments à des fins de conservation, mais aussi pour cuire - en recourant à des braises - des aliments comme de la viande ou une galette (Gélard).

La présence du sable à différents stades de procès techniques met à nouveau en relief la plasticité des statuts de cette matière: simple support de l'action technique voire du technicien, auxiliaire quand il s'agit de refroidir certaines pièces de métal en les y plongeant 
ou de polir une pièce en la frottant avec du sable, utilisé également pour remplir des bourrelets de tissus ou de cuir afin de leur donner du poids et du relief, le sable peut devenir un instrument clé de dispositifs techniques semi-industriels comme dans le cas de la dinanderie marocaine évoqué dans ce Thema par Baptiste Buob. Cet auteur met en lumière, dans le cadre de la technique ancienne de la fonte au sable à Fès et à Marrakech, le cycle de vie de la matière sable dans le processus de moulage: tantôt preneur, tantôt donneur de formes, le sable traverse les catégories de «matériau », " produit technique », « objet » et « outil », pour finalement retourner à l'état de « matière ».

Largement utilisé aujourd'hui dans la construction, le sable constitue pour les enfants du désert un formidable support d'apprentissage et de création. La reproduction en sable de l'habitation en miniature apparaît-elle ainsi comme une modalité de socialisation de l'espace mais aussi de la matière et de ses propriétés singulières (Rossie 2008). Le sable constitue par ailleurs un support fréquent de jeu, notamment au Sahara (Casajus 1988). C'est précisément le caractère éphémère des réalisations qu'il autorise qui offre un riche éventail d'expressions personnelles et permet de donner libre cours à la créativité, comme le travail de Julie Delalande l'a bien fait ressortir à propos des bacs à sable dans les cours des écoles primaires françaises (Delalande 1997). Par ailleurs, depuis les travaux fondateurs de la psychologue suisse D. M. Kalff dans les années cinquante, la thérapie par le jeu de sable s'est énormément développée en Europe et en Amérique du Nord, s'adressant aux jeunes enfants, mais aussi aux adolescents et aux adultes, et permettant, par son caractère non verbal, d'accéder à des couches profondes du psychisme en favorisant l'expression et la compréhension des troubles et des traumatismes vécus au stade préverbal de l'enfance ${ }^{8}$.

Si l'on revient aux sociétés du désert, on peut constater par conséquent la dialectique qui s'est instaurée entre les individus qui vivent dans ces régions et cette matière. Les premiers à la fois utilisent activement le sable et peuvent se prêter à l'envie à ses effets desséchants, abrasifs, absorbants, chauffants, nettoyants voire purifiants. Les effets du sable peuvent donc être recherchés ou au contraire combattus, par exemple lorsque le sable s'immisce dans les crevasses qui strient souvent la plante des pieds des éleveurs et dont ils atténuent la gêne à l'aide de beurre ou de graisse.

\section{Représentations et savoirs, hiérarchies et transmissions}

Les savoirs sur le sable, qu'il s'agisse des techniques du corps, des thérapeutiques traditionnelles (immersion, bains de sable, réduction des fractures), des utilisations culinaires du sable (Gélard), la science des traces, du langage des corps (Gagnol) témoignent de l'importance d'une ethnographie ou d'une archéologie du furtif et du disparate. En effet, les informations sur les savoirs sur le sable se retrouvent dans la littérature, mais de façon atomisée. Ceci est fort bien illustré par la contribution originale de Laurent Gagnol et son ethnographie des traces et empreintes sur le sable chez

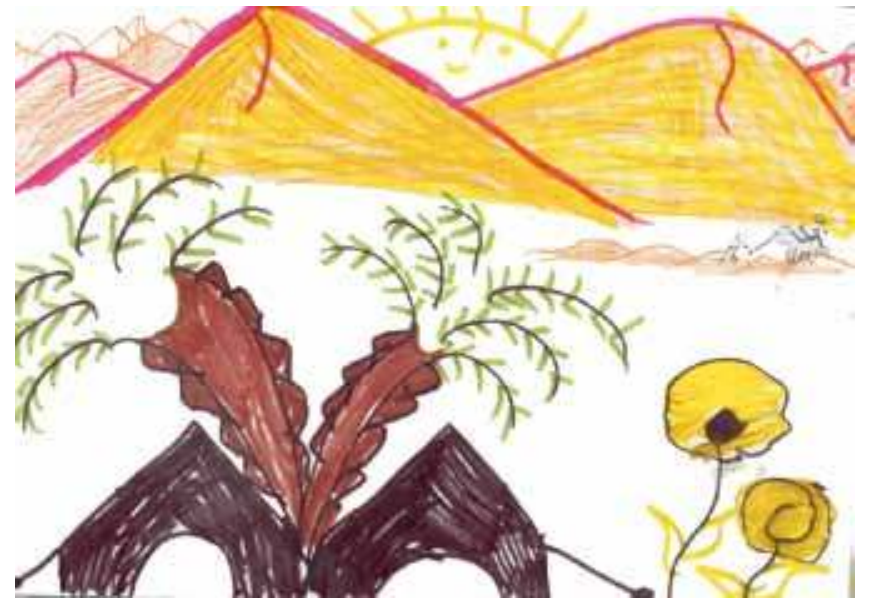


les Touaregs Kel Gress. La littérature abonde de références sur l'ingéniosité des populations pastorales à lire des traces ${ }^{9}$, mais nulle analyse approfondie n'avait jusqu'alors été menée. Il est ainsi question de la transmission des savoirs et de la manifestation des hiérarchies statutaires révélées par les connaissances des empreintes qui évoquent des allures culturelles distinctes, des manières de marcher et de se mouvoir selon, dit-on, son groupe d'appartenance (Peuls Wodaabe, Toubous...), chacun imprimant sur le sable des marques distinctes.

Toujours dans l'optique des savoirs, Catherine Taine-Cheikh propose d'aborder les représentations du sable chez les nomades maures de Mauritanie par une analyse ethno-linguistique des usages terminologiques. Usages des vocables du sable et de tout ce qui entoure la matière: dunes, vents, mondes végétal et animal. Les plantes sont nommées en fonction de l'une de leurs caractéristiques principales, telle celle qui se situe à la surface des dunes, " comme si elle nageait dans le sable». Les animaux empruntent aussi leur appellation à l'adaptation de leur physiologie au sable. Le sable est désigné également en référence à ses usages: jeu et divination.

Ce sont également les représentations du sable auxquelles Julie Misuriello consacre sa contribution, dans l'Égypte ancienne cette fois, à travers notamment une exploration de textes sacrés laissés par cette civilisation : le sable est tantôt pour les Égyptiens une contrainte et un élément redouté, tantôt une matière aux qualités exceptionnelles qui étaient associées aux valeurs de pureté, de sacralité, de protection, comme le révèlent les pratiques funéraires réservées aux princes.

On retrouve enfin une description de ces savoir-faire dans le désert occidental australien, avec la contribution de S. Poirier, où l'utilisation du bâton à fouir, possible dans un sol sableux, permet la chasse du petit gibier ou bien la récolte des végétaux (tubercules sauvages).

Les traces, empreintes et indices montrent l'importance d'une ethnographie de ces savoirs; comment ne pas songer à l'écrivain Arthur Upfield et à son personnage de Napoléon Bonaparte (Bony), ce policier rompu à toutes les astuces et subtilités de la vie dans le désert australien? Les références au sable foisonnent dans ses romans, Les sables de Windee ${ }^{10}$ ou Pas de traces dans le Bush ${ }^{11}$.

La description des savoirs sur, et à propos du sable n'a pas encore été conduite, les textes de ce numéro en proposent un aperçu qui souligne bien toute l'importance du phénomène.

\section{Sable et « modernité »}

La désertification, bien souvent provoquée aujourd'hui par des facteurs anthropiques et notamment par une surexploitation du couvert végétal (agriculture intensive, surpâturage, collecte excessive de bois mort pour produire un charbon bon marché, etc.), facilite le déplacement du sable et par voie de conséquence celui des dunes. Au Sahara, les sites de peuplement anciens sont régulièrement menacés d'ensablement et d'engloutissement, ou au contraire réapparaissent à la faveur des tempêtes de sable, offrant ainsi aux archéologues des conditions d'investigation particulières qui nécessitent une bonne dose d'opportunisme et obligent à des travaux d'urgence, mais qui permettent également un renouvellement assez fréquent de la visibilité des sites (Vernet). Dans certaines localités sahariennes, comme à Arawane dans le nord du Mali (Welland 2009: 166), des personnes - des femmes en 
l'occurrence - sont rétribuées par les habitants pour dégager les bâtiments et les points d'eau, un peu à l'image de l'héroïne du roman de Kôbô Abé La femme des sables ${ }^{12}$ dont la vie est entièrement accaparée par cette corvée, condition de sa survie et de celle de son hôte singulier. À Chinguetti, dans le Nord de la Mauritanie, la vieille ville - classée par l'Unesco au patrimoine mondial de l'Humanité en 1996 - et sa palmeraie sont, petit à petit, recouvertes par les imposants cordons dunaires qui les encerclent, comme l'a été quelques siècles plus tôt la précédente implantation de cette cité, aujourd'hui sous les sables.

Si la question de l'ensablement a depuis longtemps suscité des réflexions, elle semble se poser avec d'autant plus de gravité aujourd'hui que les projets « de développement » des hommes sont ambitieux et les moyens déployés pour « dompter » cette matière et le milieu désertique colossaux. C'est notamment le cas en Chine, où l'aire désertique s'étend à un rythme très soutenu de $200 \mathrm{~km}^{2}$ chaque mois (ibid. : 169) et où le sable fait désormais partie du paysage de certaines mégalopoles comme Pékin. Les techniques les plus connues et les plus éprouvées pour fixer les dunes consistent à y planter des essences végétales capables de retenir les amas de sable par leurs racines enchevêtrées et par les feuilles mortes qu'elles vont laisser sur le sol (Aubert de la Rüe 1940 : 123). En milieu désertique, ces efforts sont généralement concentrés autour des infrastructures qui sont directement menacées par le déplacement des dunes: en premier lieu les points d'eau, l'habitat et les routes bitumées. Ces dernières se sont multipliées au cours des dernières décennies et font régulièrement l'objet de travaux de désensablement confiés à des bulldozers. Mais elles ont également donné lieu à des projets de protection parfois assez innovants, comme celui qu'a mené l'agronome Jean Meunier au début des années 2000, en Mauritanie, et qui consistait à utiliser la force du vent pour détruire les dunes puis à les végétaliser pour les fixer.

Si les techniques sont connues, les moyens de les mettre en oeuvre font souvent défaut dans des régions où les priorités sont ailleurs et où l'on assiste, impuissants, à ce phénomène, à défaut de pouvoir « gérer » le problème sur le plus long terme. Terrains de culture

\section{Échantillons de sable}

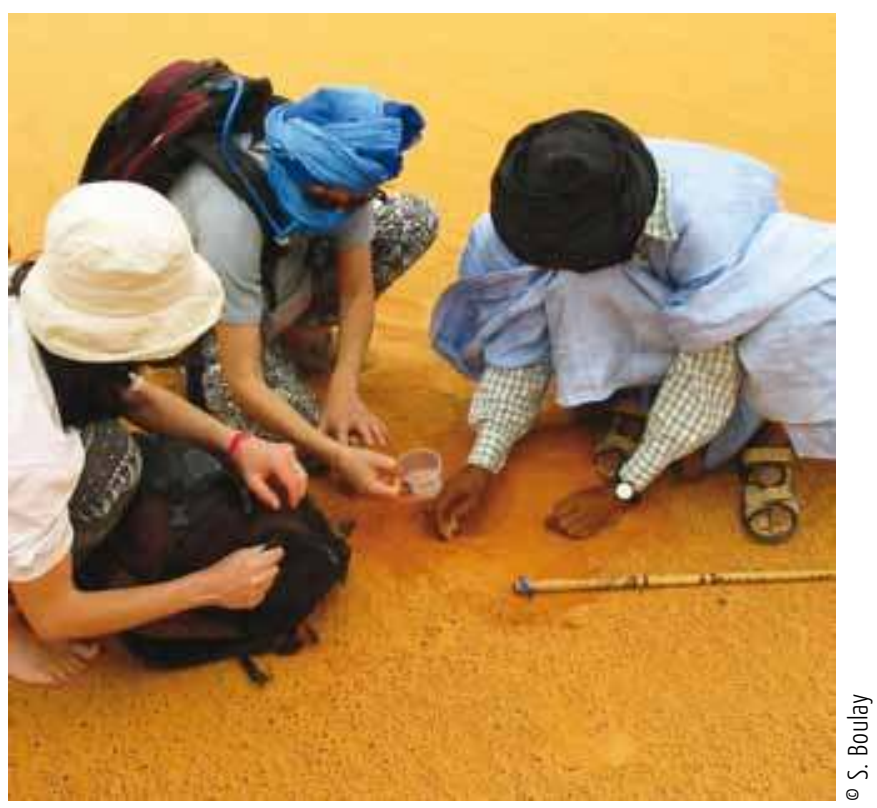


ensevelis, axes routiers traversés par des dunes, fondations de maisons emportées par le mouvement du sable, fleuves ou lacs asséchés, les infrastructures les plus coûteuses ou les plus sophistiquées ne semblent pas peser très lourd face à cette matière, comme l'illustre le documentaire photographique (Curiosa) cosigné par le géographe Roman Stadnicki et le photographe Manuel Benchetrit, consacré aux paysages urbains dans quelques grandes villes du sud de la Péninsule arabique. Dans ces espaces hypermodernes, climatisés et aseptisés, où le sable est indésirable et l'objet d'efforts de dissimulation notoires, il apparaît pourtant comme l'unique habitant d'infrastructures mégalomaniaques désertées. Trop fin pour atteindre la qualité recherchée dans le béton de construction, trop rond pour réaliser de jolies plages ou des îles artificielles dans la mer (Dubai), le sable du désert doit souvent être remplacé par du sable drainé depuis les fonds marins, voire importé d’une autre région, ce qui donne lieu à un commerce international florissant! Dans les villes modernes du désert (Pliez 2011), dont la croissance démographique semble, à première vue, permise par des conditions de vie facilitées et par un isolement moindre que par le passé, le sable met sans cesse à mal cette idéologie du progrès et de la modernisation des cadres de vie, n'empêchant pas pour autant celle-ci de continuer de prospérer et de faire rêver.

Sand painting, Herald Square, New-York, 2014

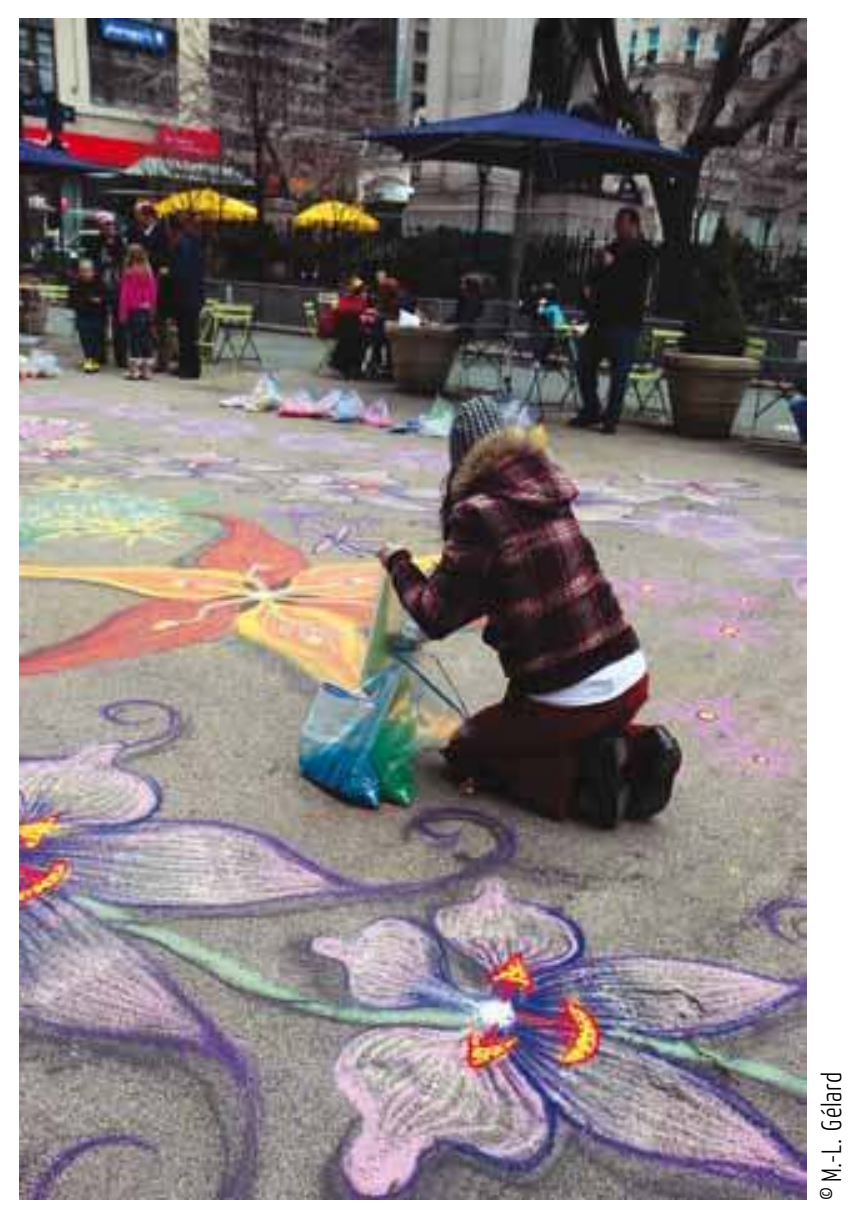




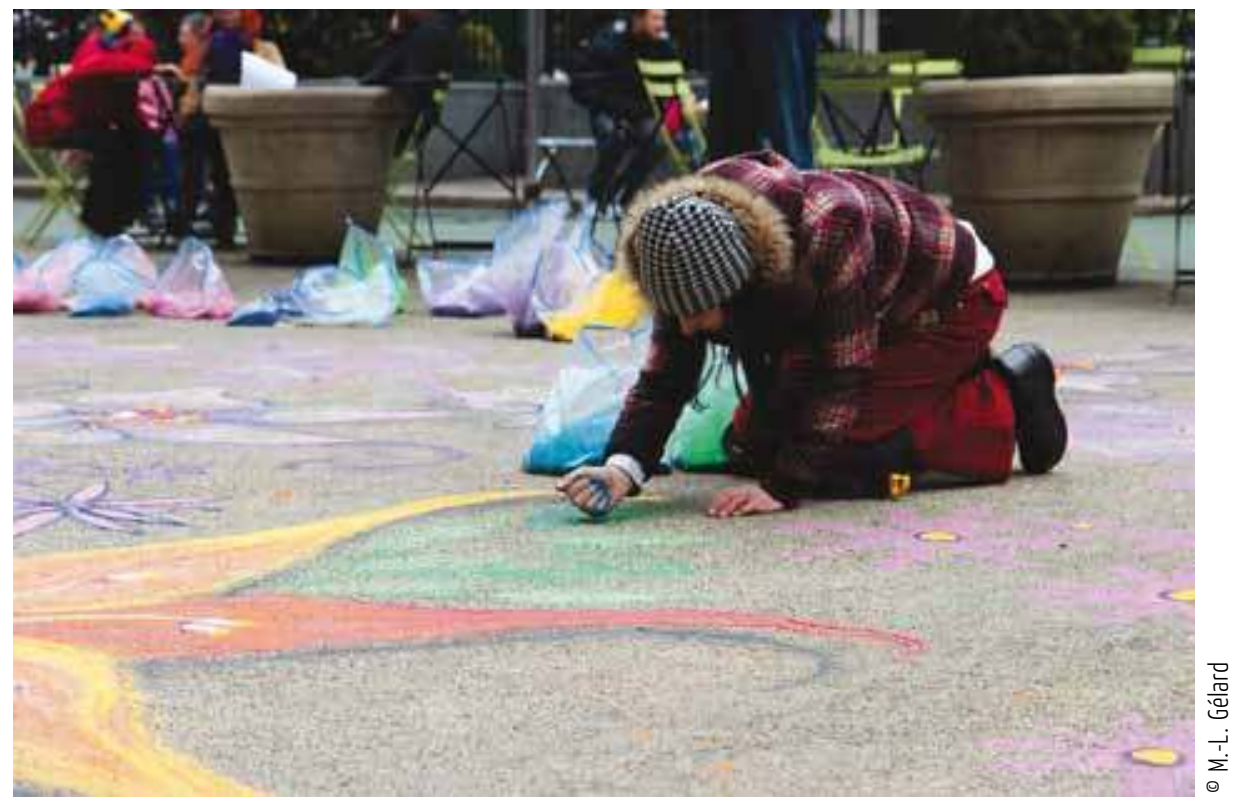

\section{NOTES}

Photo d'ouverture : Le sable de l'intérieur @ S. Boulay.

1. Soulignons l'importance de l'exploration du Sahara pour les Saint-Simoniens dont la doctrine s'oriente alors clairement vers une réunion de l'Occident et de l'Orient.

2. Il s'agit d'un phénomène connu depuis fort longtemps, autrement nommé « le chant des sables » (Thesiger 1978 : 206) et récemment étudié par une équipe de chercheurs du CNRS, B. Andreotti (2012) et S. Douady (2006). La coulée du sable se comporte comme la membrane d'un haut-parleur et produit un son, une musique singulière. Une thèse récente vient d'être consacrée à ce phénomène (Dagois 2010). Pour entendre ce fameux « chant des dunes » : http://www.youtube.com/ watch?v=t6Zt4XCHj3U (consulté le 27 novembre 2013).

3. Les collectionneurs des sables du monde sont nombreux, voire l'une des associations les plus importantes: l'AFA, Association Francophone d'Arénophilie: http:// www.la-passion-des-sables.net/spip/ (consulté le 27 novembre 2013).

4. Le terme arabe « șahrầ' », qui a donné le nom Sahara, est le féminin de l'adjectif « așḥar », qui désigne la couleur fauve (Callot 1995).

5. Voir l'intéressante analyse de l'auteur sur les étendues de sable apparues dans les grandes villes à la création de plages artificielles et l'étude de l'impact de ces tonnes de sable déversées.

6. Voir la belle réalisation en ligne: http://www.youtube.com/ watch?v=KEzyzM6MMmg (consultéle 27 novembre 2013).
7. « En effet, ne pas lever le doigt confère au tracé une propriété correspondant à ce que les mathématiciens appellent un graphe eulérien $»$ (Chemillier 2004 : 272).

8. Pour découvrir les travaux de la société internationale de thérapie par le sable: www.isst-society.com et la revue éditée par sa branche américaine: http://www.sandplay.org/store/products.php?catid=3\&category=Journal (pages consultées le 27 novembre 2013).

9. Citons Thesiger (1978: 77) « Ici, tout homme connaissait les empreintes de ses propres bêtes et certains étaient capables d'identifier les traces de presque tous les chameaux qu'ils avaient rencontrés. D'un simple coup d'œil, ils pouvaient dire, d'après la profondeur des empreintes, si un chameau était monté, ou non, si une chamelle était pleine ou non. En étudiant des traces inconnues, ils pouvaient déterminer l'endroit d'où venait l'animal. Les chameaux originaires des Sables, par exemple, ont la sole si tendre que les lambeaux de peau s'en détachent, tandis que ceux qui viennent des plaines de gravier ont la sole lisse et polie ».

10. «Il resta quelques instants silencieux pendant qu'il traçait un plan grossier sur le sol de sable rouge avec un petit bâton. [...] Tout autour, le paysage est un labyrinthe de basses crêtes de sable sur lesquelles poussent des pins et quelques mulgas. [...] La nature a effacé toutes les traces et a eu largement le temps d'enfouir tous les indices profondément dans le sable. »(Upfield 1994 : 16, 17 et 21). 
11. Roman policier ethnologique, écrit en 1940 par Arthur Upfield qui met à l'honneur, notamment par l'ingéniosité des savoirs sur les traces, une culture aborigène alors méprisée.
12. Roman adapté au cinéma en 1964 par le cinéaste japonais Teshigahara en collaboration avec le romancier.

\section{RÉFÉRENCES}

Abé, K. 1967 La Femme des sables. Paris: Stock.

Andreotti, B. 2012 Sonic Sands, Reports on Progress in Physics 75 (2) [en ligne] : http://www.pmmh.espci. fr/fr/morphodynamique/papers/A55_0034-4885_75_2_026602.pdf consulté le 14 novembre 2013, doi :10.1088/0034-4885/75/2/026602.

Ascher, M. 1998 Mathématiques d'ailleurs. Paris: Seuil.

Aubert de la Rüe, E. 1940 L'Homme et le vent. Paris: Gallimard.

Augé, M. 1995 Un ethnologue à la plage, Le Monde Diplomatique, août 1995.

Bagnold, R. A. 1954 The Physics of Blown Sand and Desert Dunes. London: Methuen.

Bahti, M., Baatsoslanii, E. 2000 A guide to Navajo sandpaintings. Tucson: Rio Nuevo Publishers.

Cabane, J.-P. 1995 Les Dessins sur sable à Vanuatu, un art de la «parole ». In F. Angleviel, Parole, communication et symbole en Océanie. Paris: L'Harmattan: 271-304.

Callot, Y. 1995 « șaḥrâ'» in Encyclopédie de l'Islam. Leiden: E. J. Brill: 875-876.

Camps, G. 1998 « Four » Encyclopédie Berbère (19). Aix-en-Provence: Édisud: 2922-2926.

Caratini, S. 2009 La Dernière marche de l'empire. Une éducation saharienne. Paris: Les empêcheurs de penser en rond/La Découverte.

Casajus, D. 2009 Charles de Foucauld. Moine et savant. Paris: CNRS.

- 2007 Henri Duveyrier. Un Saint-Simonien au désert. Paris: Ibis Press.

- 2006 Édition du Journal d'un voyage dans la province d'Alger d'Henri Duveyrier. Paris: Édition des Saints Calus.

— 1988 Jeux touaregs de la région d'Agadez, Journal des Africanistes 58 (1): 23-49.

Chemillier, M. 2004 Représentations musicales et représentations mathématiques, L'Homme (171-172): 267-284.

Clarkson, B. 1999 Considérations historiques et contextualisation de la recherche sur les géoglyphes au Chili, Anthropologie et Sociétés 23 (1): 125-150.

Corbin, A. 1995 L'Avènement des loisirs 1850-1960. Paris: Aubier.

Côte, M. 2012 Signatures sahariennes. Terroirs et territoires vus du ciel. Aix-en-Provence: Presses Universitaires de Provence.

Dagois, S. 2010 Le Chant des dunes: mouvements collectifs dans un écoulement granulaire. Paris 7: thèse de physique acoustique, sous la direction de S. Douady.

Deacon, B. 1934 Geometrical Drawings from Malekula and Other Islands of the New Hebrides, Journal of the Royal Anthropology Institute (64): 129-175.

Delalande, J. 1995 Le Sable doux, la chaîne et le plouf-plouf. Vers une anthropologie de l'enfant, Ethnologie Française XXV, 4 : 617- 628.

Devienne, E. 2011 Des plages dans la ville. Los Angeles et la réflexion sur la plage urbaine idéale, La vie des idées [en ligne]: http://www.laviedesidees.fr/Des-plages-dans-la-ville.html consulté le 14 novembre 2013.

Douady, S., Manning, A., Hersen, $E$ al. 2006 The song of the dunes as a self-synchronized instrument, Physical Review Letters 97 http://link.aps.org/doi/10.1103/PhysRevLett.97.018002 consulté le 14 novembre 2013, doi :10.1103/PhysRevLett.97.018002.

Duveyrier, H. 1864 Les Touaregs du Nord. Paris: Challamel.

- 1863 Note sur les Touaregs et leur pays. Paris: Imprimerie de L. Martinet.

Enfantin, P. 1843 Colonisation de l'Algérie. Paris: P. Bertrand. 
Foucauld de, C. 1998 Correspondances sahariennes. Lettres inédites aux pères blancs et aux sceurs blanches. Paris: Éditions du Cerf.

- 1986 Carnets de Tamanrasset. Paris: Nouvelle Cité.

- 1888 Reconnaissance au Maroc, 1883-1884. Paris: Éditions géographiques, maritimes et coloniales.

Fromentin, E. 1857 Un été au Sahara. Paris: Michel Lévy frères.

Gast, M. 1968 Alimentation des populations de l'Ahaggar. Étude ethnographique. Paris: Arts et Métiers Graphiques.

Gerdes, P. 1995 Une tradition géométrique en Afrique, les dessins sur le sable. Paris: L'Harmattan.

Gibson, James J. 1979 The Ecological Approach to Visual Perception. Boston: Houghton Mifflin.

Ginzburg, C. 1989 Mythes, emblèmes, traces. Morphologie et histoire. Paris: Flammarion.

Ingold, T. 2007 Materials Against Materiality, Archeological Dialogues 14 (1): 1-16.

Julien, M.-P. \& Warnier, J.-P. (dir.) 1999 Approches de la culture matérielle, Corps à corps avec l'objet. Paris: L'Harmattan.

Kilani, M. 2000 L'Invention de l'autre. Essais sur le discours anthropologique. Lausanne: Payot.

Lemonnier, P. (dir.) 1993 Technological choices, Transformation in material cultures since the Neolithic. London et New York: Routledge.

Leroi-Gourhan, A. 1992 [1943] L'Homme et la matière. Paris: Albin Michel.

Martinelli, B. (dir.) 2005 L'Interrogation du style, Anthropologie, techniques et esthétique. Aix-en-Provence: Presses de l'Université de Provence.

Mauss, M. 1997 [1936] Les Techniques du corps. In M. Mauss. Sociologie et anthropologie. Paris: PUF.

Monod, T. 1937 Méharées, explorations au vrai Sahara. Paris: Éditeur Je Sers.

Morel, A. 2008 Milieux et Paysages du Sahara. Paris: Ibis Press.

Newcomb, F. J., Reichard, G. A 1975 Sandpaintings of the Navajo Shooting Chant. New York: Dover Publications.

Pliez, O. 2011 Les Cités du désert. Des villes sahariennes aux saharatowns. Toulouse et Marseille: Presses universitaires du Mirail et Institut de recherche pour le développement.

Pouillon, F. 2006 Dictionnaire des orientalistes de langue française. Paris: IISMM, Karthala.

Rey-Debove, J. \& Rey, A. (dir.) 2013 Le Petit Robert. Dictionnaire alphabétique et analogique de la langue française. Paris: Le Robert.

Rossie, J.-P. 2008 Cultures Ludiques Sahariennes et Nord-Africaines. La vie domestique dans les jeux et jouets. Stockholm: SITREC.

Soleilhavoup, F. 2010 Curiosités géologiques au Sahara. Paris: Ibis Press.

Therrien, M. Traces sur la neige, signes sur le papier. Significations de l'empreinte chez les Inuit Nunavimmiut (Arctique québécois), Journal de la Société des Américanistes 76: 33-53.

Thesiger, W. 1978 Le Désert des Déserts. Avec les Bédouins derniers nomades de l’Arabie du Sud. Paris: Plon.

- 1959 Arabians Sands. Londres: Penguin.

Upfield, A. [1940], 1994 Pas de traces dans le bush. Paris: 10/18.

— [1931], 1994 Les Sables de Windee. Paris: 10/18.

Urbain, I. [1860], 2000 L’Algérie pour les Algériens. Paris: Seguier.

Vadrot, Ph. 2006 La Neige dans tous ses états: un métissage d'imaginaires de la glisse et de la forme, ethnographiques.org 10 [en ligne] : http://ethnographiques.org/2006/Vadrot consulté le 14 novembre 2013.

Varichon, A., Roccella, C. 2006 Ettre sable. Paris: Seuil.

Warnier, J.-P. 1999 Construire la culture matérielle: l'homme qui pensait avec ses doigts, Paris: PUF.

Welland, M. 2009 Sand. The Never-ending Story. Berkeley-Los Angeles: University of California Press.

\section{POUR CITER CET ARTICLE}

Boulay, S., Gélard, M.-L. 2014 Vivre le Sable! Corps, matière et sociétés: une introduction, in S. Boulay \& M.-L. Gélard, Vivre le sable! Corps, matière et sociétés, Techniques \& Culture 61: 10-27. 